\title{
Kissing catheter technique for percutaneous catheter drainage of necrotic pancreatic collections in acute pancreatitis
}

\author{
PANKAJ GUPTA $^{1}$, SUZANNE KOSHI ${ }^{1}$, JAYANTA SAMANTA ${ }^{2}$, HARSHAL MANDAVDHARE ${ }^{2}$, \\ VISHAL SHARMA $^{2}$, SAROJ K SINHA ${ }^{2}$, USHA DUTTA ${ }^{2}$ and RAKESH KOCHHAR ${ }^{2}$ \\ Departments of ${ }^{1}$ Radiodiagnosis and Imaging, and ${ }^{2}$ Gastroenterology, Nehru Hospital, \\ Postgraduate Institute of Medical Imaging and Research, Chandigarh, Punjab 160012, India
}

Received November 4, 2019; Accepted April 29, 2020

DOI: $10.3892 /$ etm. 2020.8897

\begin{abstract}
One of the critical factors for predicting the success of percutaneous catheter drainage (PCD) is the mean CT density of collection. A higher CT density suggests more necrotic solid tissue within the collection. In the present study, a novel technique for PCD of the necrotic pancreatic collection with a higher mean CT density was evaluated. It was a retrospective study of patients with acute pancreatitis (AP) who underwent PCD of pancreatic collections between May 2018 and December 2018. Patients with pancreatic collections having a CT density of $>30$ Hounsfield Units (HU) were considered for PCD using the kissing catheter technique. This technique involved placing two catheters side-by-side through a single cutaneous entry site, as the conventional technique of PCD may not be effective. The technical details, outcomes and complications of this technique were recorded. A total of 10 patients with a mean age of 30 years underwent PCD using this technique. All patients had severe pancreatitis with a mean CT severity index of 9 (range, 8-10). The mean CT density was $37 \mathrm{HU}$ (range, 32-56). Successful management with PCD alone was achieved in 8 patients. The other 2 patients underwent surgical necrosectomy. One patient who underwent surgical necrosectomy died. Minor complications occurred in 3 patients. The kissing catheter technique allows for a higher success rate of PCD compared with that of the conventional method of PCD, in collections with a higher mean CT density.
\end{abstract}

Correspondence to: Dr Pankaj Gupta, Department of Radiodiagnosis and Imaging, A-Block, Nehru Hospital, Postgraduate Institute of Medical Imaging and Research, Sector 12, Chandigarh, Punjab 160012, India

E-mail: pankajgupta959@gmail.com

Abbreviations: PCD, percutaneous catheter drainage; CTSI, CT severity index; F, French; ICU, intensive care unit

Key words: acute pancreatitis, percutaneous catheter drainage, collections

\section{Introduction}

Percutaneous catheter drainage (PCD) is an integral part of the management of necrotic pancreatic collections in the setting of acute pancreatitis (AP). The PANTER trial demonstrated that a minimally invasive step-up approach utilizing percutaneous catheter followed by minimally invasive retroperitoneal necrosectomy reduced the rate of major complications as well as mortality when compared to open necrosectomy (1). Among the patients assigned to the step-up protocol, $35 \%$ of patients were able to be treated with catheter drainage alone. Certain studies have reported a success rate of PCD alone of as high as $50-60 \%$ (2-4). In addition, a proactive or aggressive protocol of PCD is associated with better outcomes (5-8). The presence of solid, necrotic contents within the collection, as suggested by a higher mean CT density of collection, is associated with a lower success of PCD (9-11). The present study proposed a modification of the PCD technique, the 'kissing catheter' technique, to achieve successful drainage of these collections. This technique involves placing two catheters side-by-side through a single cutaneous entry site. The aim is to allow aggressive flushing (through one catheter) and aspiration (through the other catheter) and remove as much necrotic tissue as possible instead of just draining the fluid component of the pancreatic collection.

\section{Materials and methods}

Patients and requirement for PCD. The present study was a retrospective evaluation of a prospectively acquired database of patients with AP who underwent PCD of necrotic pancreatic collections (acute necrotic collections/walled-off necrosis) at the Postgraduate Institute of Medical Education and Research, Chandigarh, between May and December 2018. Patients who underwent PCD with the modified technique (kissing catheter technique) were included for analysis. The present study was approved by the institutional ethics committee of the Postgraduate Institute of Medical Education and Research, Chandigarh. As this was a retrospective study, informed consent for inclusion in a study was not required from the patients, but patients had given procedural consent. The diagnosis of AP was based on the revised Atlanta criteria (12). Patients underwent an initial contrast-enhanced CT between 3 and 7 days of pain 


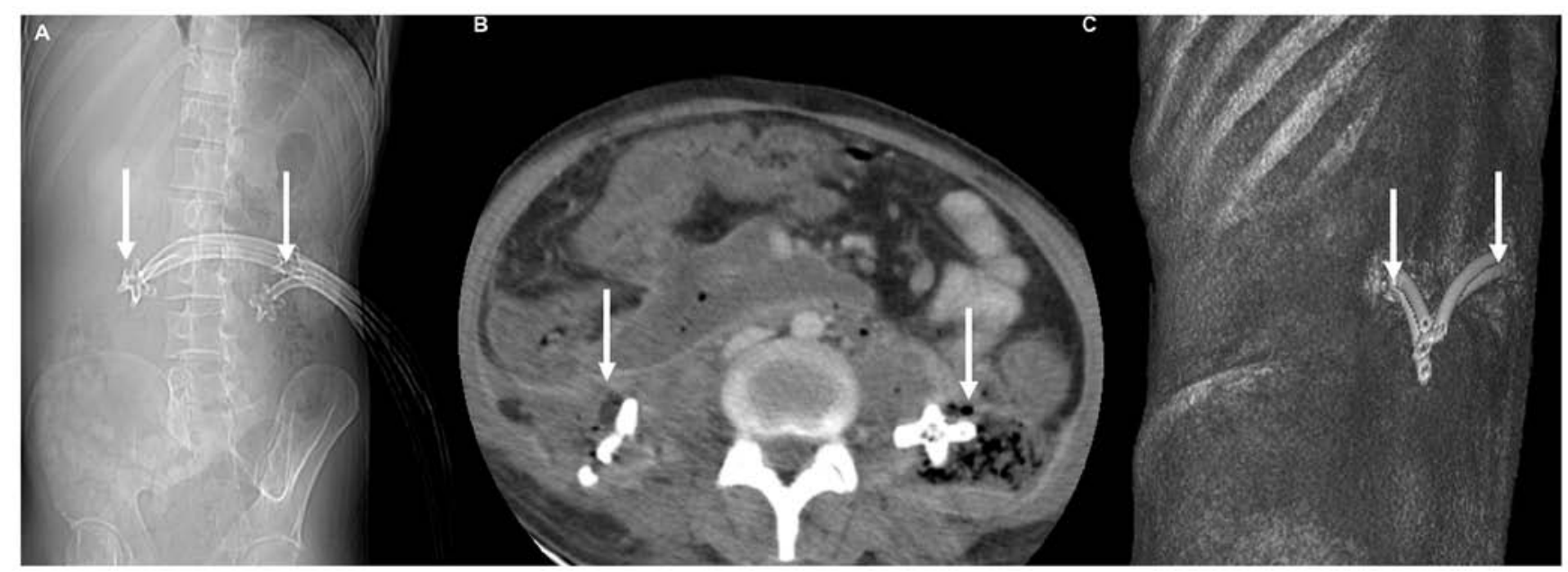

Figure 1. Images of a 22-year-old male with alcohol-induced acute pancreatitis with bilateral paracolic gutter collections managed with kissing catheter technique. (A) CT Scanogram displaying two catheters placed side-by-side in bilateral flanks (arrows). (B) Axial contrast-enhanced CT and (C) volume-rendered images from the CT study reveal the catheters placed in bilateral paracolic gutters through a single cutaneous opening (arrows).

onset. A modified CT severity index (CTSI) was assigned. The mean CT density of the collection was calculated by placing a circular region of interest over multiple parts of the collection and taking a mean value. The indications of the drainage were recorded.

Technical details. PCD was performed by an interventional radiologist $(\mathrm{PG})$ with five years' experience in performing abdominal interventions using ultrasound or CT guidance. Catheter insertion was performed using the Seldinger technique. Following the initial image-guided puncture of the collection, a 0.035" stiff guidewire (Amplatz Extra Stiff Wire Guide; Cook Medical) was placed into the collection. After serial dilatations of the access tract using stiff fascial dilators (Devon ${ }^{\circledR}$ Innovations Private Ltd.), a single 12 or 14 French (F) catheter (Biomedical Health care, India) was placed into the collection. Patients were re-assessed for the resolution of organ failure and sepsis. A repeat ultrasound was performed after three days to identify any residual collection and determine the amount of solid debris. If a patient had persistent or worsening organ failure and ongoing sepsis and CT indicated a $<50 \%$ reduction in the size of the collection and attenuation $>30$ Hounsfield Units (HU), they were subjected to the kissing catheter technique (11).

Details of the kissing catheter technique. A total of two 0.035" stiff guidewires were placed through the pre-existing catheter into the collection. The catheter was then removed. Over one of the guidewires, a new $14 \mathrm{~F}$ catheter was placed. Over the other guidewire, a 10-14 F catheter was placed after fascial dilatation. Immediately following the placement of the two catheters, flushing with normal saline and active aspiration was performed by using one of the catheters for flushing and the other catheter for active aspiration. The endpoint of flushing was a clear aspirate from the two catheters without any visible solid component. The flushing and aspiration were performed daily by the interventional radiologist until the follow-up CT scan indicated complete resolution of the collection and the organ failure and sepsis were resolved. During this period, upsizing of each catheter (16-24 F) was also performed based on the size of the collection. After achieving resolution of the collection and until the catheters had an output of $<20 \mathrm{ml} / \mathrm{day}$, the catheters were clamped for 2 days and a repeat ultrasound was performed. If there was no clinical deterioration, pericatheter leakage or residual collection, the catheters were removed. The patients were subjected to surgical necrosectomy if the collection persisted even after three upsizing procedures (after the insertion of two catheters) and organ failure or sepsis persisted.

Follow-up. Following catheter removal, patients were assessed every week by the interventional radiologist for the 1st month after discharge from the hospital. The following technical and outcome parameters were recorded: The interval from onset of pain to application of the kissing catheter technique, the total size of the catheter combination, duration of catheter drainage, complications, requirement for surgery, length of hospital stay, length of intensive care unit (ICU) stay and death.

\section{Results}

Baseline characteristics. A total of 70 patients with moderately severe and severe AP were managed with PCD during the study period. A total of 10 patients (14.2\%) with a mean CT density of the pancreatic collections of $>30 \mathrm{HU}$ who did not respond to the conventional PCD method were subjected to the kissing catheter technique. The cohort comprised 7 males and 3 females. The mean age was 30 years (range, 17-45 years). The aetiology of AP was alcohol abuse $(n=4)$, gallstones $(n=4)$ and idiopathic causes $(n=2)$. All the patients treated with this technique had severe AP. The CTSI ranged from 8-10 (mean CTSI 9) and the largest dimensions of the collections ranged from $10-20 \mathrm{~cm}$ (mean size, $14.5 \mathrm{~cm}$ ). The sites of the collection were paracolic gutter $(n=6)$ and lesser sac $(n=4)$. The mean CT density was $37 \mathrm{HU}$ (range, 32-56).

Details of PCD. The indications of the initial PCD were suspected infection $(n=6)$, persistent or worsening organ failure $(n=3)$ and intra-abdominal hypertension $(n=1)$. Intra-abdominal 


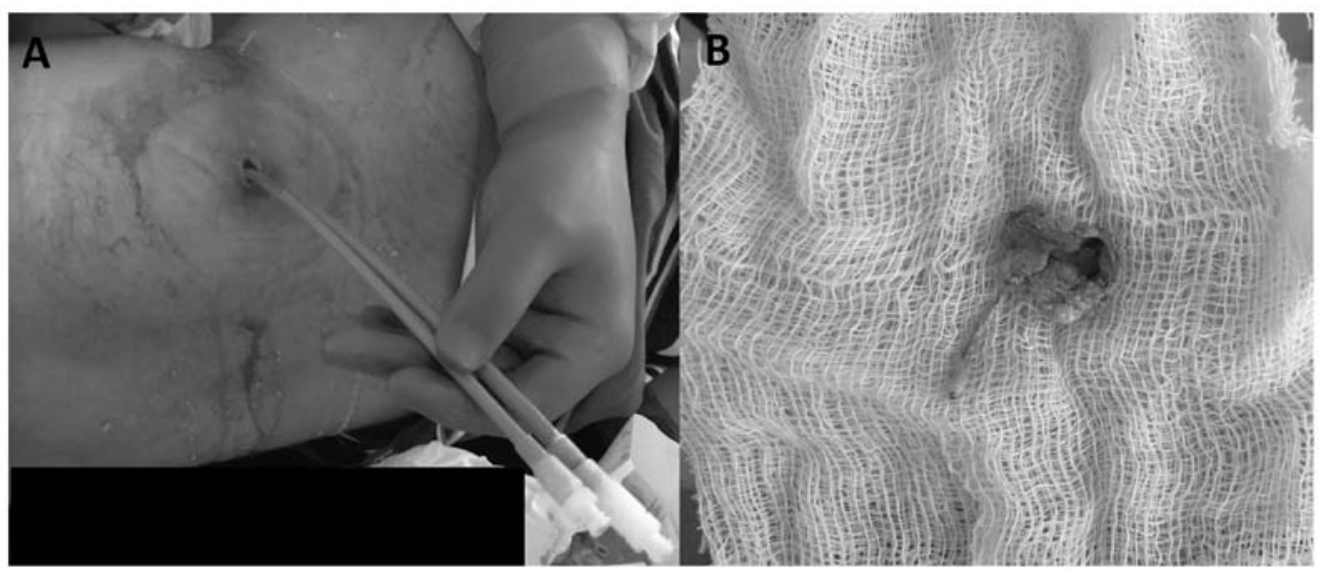

Figure 2. Clinical images of the entry site of the kissing catheter and the necrotic debris. (A) Abdominal image of the patient, a 30-year-old male with alcohol-induced acute pancreatitis, subjected to percutaneous catheter drainage with the 'kissing catheter' technique; the two $14 \mathrm{~F}$ catheters placed side-by-side in the left paracolic gutter are displayed. (B) Image of the solid necrotic debris (measuring $4 \times 2.8 \mathrm{~cm}$ ) removed using this technique.

hypertension was defined by an intra-abdominal pressure of $>12 \mathrm{mmHg}(13)$. The mean interval between the onset of pain and PCD was 13.7 days (range, 7-26 days). The mean interval between the initial PCD and the kissing catheter technique was 7.6 days (range, 3-14 days). The mean maximum size of the catheter combination was $33.6 \mathrm{~F}$ (range, 28-48 F). The first catheter cultures were positive in 6 patients. Images of a representative case are provided in Figs. 1 and 2. The complications associated with this technique were catheter dislodgement $(n=2)$, external pancreatic fistula $(n=1)$ and bleeding from the catheter site $(n=1)$. Catheter dislodgement was managed by placing new catheters through the pre-existing tract. The external pancreatic fistula resolved after transpapillary pancreatic stenting, while bleeding from the catheter site required no active intervention. The mean total duration of the PCD was 28.6 days (range, 12-43 days). None of the patients underwent endoscopic drainage.

Clinical outcomes. The mean length of hospital stay was 32.4 days (range, 10-59 days). A total of 3 patients required ICU admission. The mean duration of ICU stay was 17 days (range, 11-45 days). Furthermore, 2 patients underwent surgical necrosectomy, of which 1 patient died. Table I provides the technical details and outcome parameters for all of the patients.

\section{Discussion}

Necrotic fluid collection is the most critical local complication of AP $(14,15)$. An infected pancreatic collection is responsible for significant morbidity and mortality associated with acute necrotizing pancreatitis (1). Open necrosectomy allows for effective removal of the necrotic material and was previously the treatment of choice for necrotic collections (16). However, it is associated with significant morbidity and mortality (1). Image-guided percutaneous drainage of pancreatic collections was initially advocated by Freeny et al (17) in 1998. Multiple other studies have reported high treatment success and lower morbidity and mortality with the PCD (18-22). The landmark PANTER trial in 2010 compared primary necrosectomy to a 'step-up approach' in patients with infected necrotizing pancreatitis (1). In this approach, catheter drainage (percuta- neous or endoscopic) was the initial step. In patients who did not improve with catheter drainage underwent video-assisted retroperitoneal drainage, and if required, open necrosectomy. This trial indicated that $35 \%$ of patients could be treated with catheter drainage alone. The high success rates of PCD has also been confirmed in the two recent meta-analyses $(3,9)$.

One of the most important factors predicting the failure of PCD is the amount of necrotic tissue within the collection, as determined by the higher mean CT density and heterogeneity (9-11). The catheter drainage protocol adopted in various published studies is effective in draining the fluid component of the collection (17-22). However, this PCD protocol is not efficient for collections that contain a large amount of necrotic tissue. Certain studies have reported that a proactive PCD protocol has increased efficacy in treating pancreatic collections (5-8). In all the published studies, a single percutaneous catheter is placed in a collection. Active flushing and aspiration, integral to the success of PCD, are challenging to achieve with a single catheter, particularly in collections with a large amount of solid component. The kissing catheter technique described in the present study uses two catheters in combination, placed through a single cutaneous opening. The two catheters also form a closed system for flushing and active aspiration that allows for the removal of necrotic debris. This approach prevents catheter blockade. It may be argued that two catheters placed into the collection through different sites or a single large catheter may function equally well. The placement of two catheters through different sites may be difficult given a limited acoustic window or bowel-free approach for lesser sac collections. On the other hand, the placement of a single large catheter does not allow for aggressive flushing and aspiration. The mean interval from the onset of pain to PCD was 13.7 days. Although it is preferable to delay the interventions for pancreatic collections as far as possible, recent studies have indicated comparable outcomes of PCD for acute necrotic collections and walled-off necrosis $(4,8)$. A trial comparing postponed vs. early drainage of infected necrotic collections (POINTER trial) is being conducted by the Dutch pancreatitis group (23).

Potential complications of the technique include the development of external pancreatic fistula and increased risk of vascular and bowel injury due to large catheter sizes $(24,25)$. 


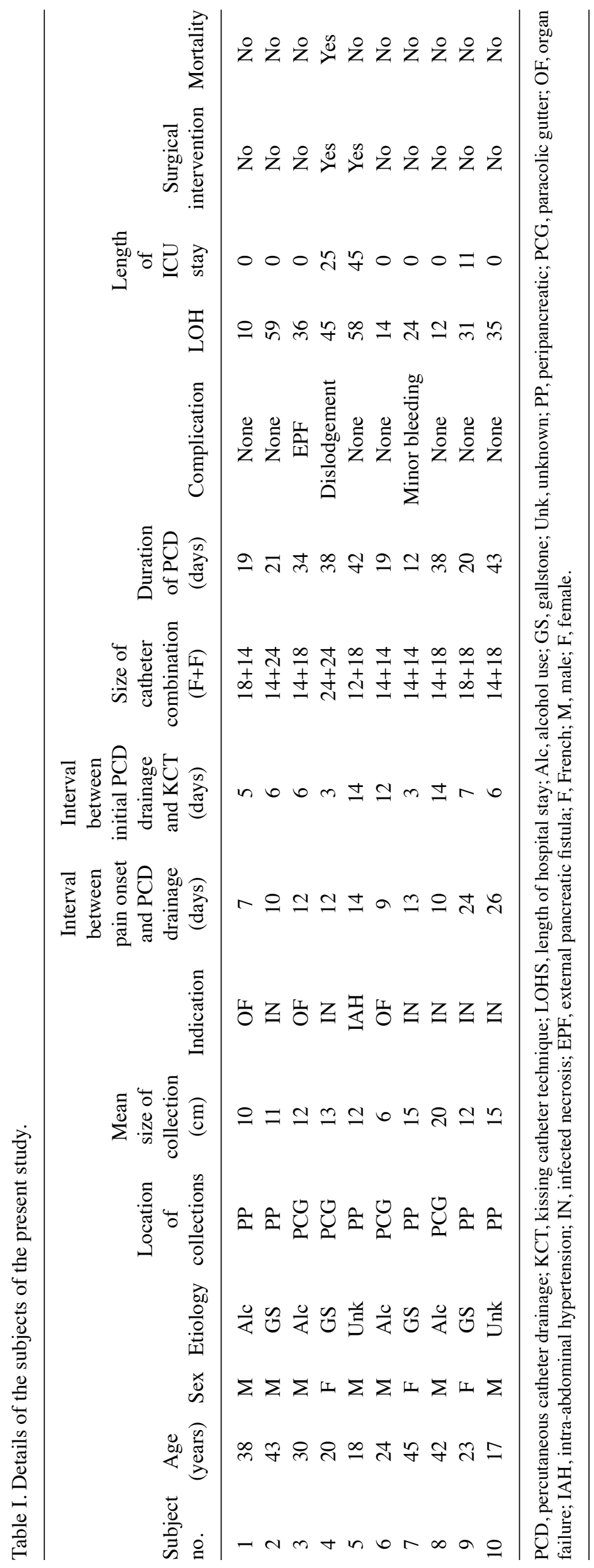


Even though one patient developed external pancreatic fistula, none of the patients suffered any vascular or bowel injury. Additional concerns include skin excoriation and infection (26). This mandates meticulous care of skin with skin hygiene, regular dressing and use of emollients.

There are a few limitations to the present study. First, the sample size was small. However, the present study aimed to evaluate the novel PCD method. The outcomes and complications of the kissing catheter technique were not compared with the standard method of PCD. This was not possible given the small size of the cohort.

In conclusion, the kissing catheter technique has the potential to further improve the success rate of PCD of necrotic collections containing a large amount of necrotic tissue. Randomized controlled trials are required to confirm the utility of this novel technique in the definitive management of necrotic pancreatic collections.

\section{Acknowledgements}

Not applicable.

\section{Funding}

Not applicable.

\section{Availability of data and materials}

All data generated or analyzed during this study are included in this published article.

\section{Authors' contributions}

PG performed the interventions, $\mathrm{PG}$ and SK analyzed the data and wrote the manuscript. JS, HM, VS, SKS, UD and RK were involved in clinical care of patients and revised the manuscript. All authors read and approved the final manuscript.

\section{Ethics approval and consent to participate}

The present study was approved by the institutional ethics committee of the Postgraduate Institute of Medical Education and Research, Chandigarh. As this was a retrospective study, informed consent for inclusion in a study was not required from the patients, but patients had given procedural consent.

\section{Patient consent for publication}

Not applicable.

\section{Competing interests}

The authors declare that they have no competing interests.

\section{References}

1. van Santvoort HC, Besselink MG, Bakker OJ, Hofker HS, Boermeester MA, Dejong $\mathrm{CH}$, van Goor H, Schaapherder AF, van Eijck CH, Bollen TL, et al; Dutch Pancreatitis Study Group: A step-up approach or open necrosectomy for necrotizing pancreatitis. N Engl J Med 362: 1491-1502, 2010.
2. Ke L, Li J, Hu P, Wang L, Chen $\mathrm{H}$ and Zhu Y: Percutaneous Catheter Drainage in Infected Pancreatitis Necrosis: A Systematic Review. Indian J Surg 78: 221-228, 2016.

3. van Baal MC, van Santvoort HC, Bollen TL, Bakker OJ, Besselink MG and Gooszen HG; Dutch Pancreatitis Study Group: Systematic review of percutaneous catheter drainage as primary treatment for necrotizing pancreatitis. Br J Surg 98: $18-27,2011$

4. Mallick B, Dhaka N, Gupta P, Gulati A, Malik S, Sinha SK, Yadav TD, Gupta V and Kochhar R: An audit of percutaneous drainage for acute necrotic collections and walled off necrosis in patients with acute pancreatitis. Pancreatology 18: 727-733, 2018.

5. van Grinsven J, Timmerman P, van Lienden KP, Haveman JW, Boerma D, van Eijck CH, Fockens P, van Santvoort HC, Boermeester MA and Besselink MG; Dutch Pancreatitis Study Group: Proactive Versus Standard Percutaneous Catheter Drainage for Infected Necrotizing Pancreatitis. Pancreas 46: 518-523, 2017.

6. Sugimoto M, Sonntag DP, Flint GS, Boyce CJ, Kirkham JC, Harris TJ, Carr SM, Nelson BD, Bell DA, Barton JG, et al: Better Outcomes if Percutaneous Drainage Is Used Early and Proactively in the Course of Necrotizing Pancreatitis. J Vasc Interv Radiol 27: 418-425, 2016.

7. Sugimoto M, Sonntag DP, Flint GS, Boyce CJ, Kirkham JC, Harris TJ, Carr SM, Nelson BD, Barton JG and Traverso LW: A percutaneous drainage protocol for severe and moderately severe acute pancreatitis. Surg Endosc 29: 3282-3291, 2015.

8. Gupta P, Gupta J, Kumar C, Samanta J, Mandavdhare H, Sharma V, Sinha SK, Gupta V, Yadav TD, Dutta U, et al: Aggressive Percutaneous Catheter Drainage Protocol for Necrotic Pancreatic Collections. Dig Dis Sci: Feb 5, 2020 (Epub ahead of print). doi: 10.1007/s10620-020-06116-6.

9. Hollemans RA, Bollen TL, van Brunschot S, Bakker OJ, Ahmed Ali U, van Goor H, Boermeester MA, Gooszen HG, Besselink MG and van Santvoort HC; Dutch Pancreatitis Study Group: Predicting Success of Catheter Drainage in Infected Necrotizing Pancreatitis. Ann Surg 263: 787-792, 2016.

10. Tong Z, Li W, Yu W, Geng Y, Ke L, Nie Y, Sun J, Ni H, Wang X, Ye X, et al: Percutaneous catheter drainage for infective pancreatic necrosis: Is it always the first choice for all patients? Pancreas 41: 302-305, 2012.

11. Guo Q, Li A and $\mathrm{Hu}$ W: Predictive factors for successful ultrasound-guided percutaneous drainage in necrotizing pancreatitis. Surg Endosc 30: 2929-2934, 2016.

12. Banks PA, Bollen TL, Dervenis C, Gooszen HG, Johnson CD, Sarr MG, Tsiotos GG and Vege SS; Acute Pancreatitis Classification Working Group: Classification of acute pancreatitis--2012: Revision of the Atlanta classification and definitions by international consensus. Gut 62: 102-111, 2013.

13. Malbrain ML, Cheatham ML, Kirkpatrick A, Sugrue M, Parr M, De Waele J, Balogh Z, Leppäniemi A, Olvera C, Ivatury R, et al: Results from the International Conference of Experts on Intra-abdominal Hypertension and Abdominal Compartment Syndrome. I. Definitions. Intensive Care Med 32: 1722-1732, 2006.

14. Gupta P, Jain R, Koshi S, Gulati A, Samanta J, Mandavdhare H, Sharma V, Sinha SK, Dutta U, Sandhu MS, et al: Radiation dose from computed tomography in patients with acute pancreatitis: An audit from a tertiary care referral hospital. Abdom Radiol (NY) 45: 1517-1523; Epub ahead of print, 2020.

15. Gupta P, Rana P, Bellam BL, Samanta J, Mandavdhare H, Sharma V, Sinha SK, Dutta U and Kochhar R: Site and size of extrapancreatic necrosis are associated with clinical outcomes in patients with acute necrotizing pancreatitis. Pancreatology 20: 9-15, 2020 .

16. Beger HG, Büchler M, Bittner R, Oettinger W, Block S and Nevalainen T: Necrosectomy and postoperative local lavage in patients with necrotizing pancreatitis: Results of a prospective clinical trial. World J Surg 12: 255-262, 1988.

17. Freeny PC, Hauptmann E, Althaus SJ, Traverso LW and Sinanan M: Percutaneous CT-guided catheter drainage of infected acute necrotizing pancreatitis: Techniques and results. AJR Am J Roentgenol 170: 969-975, 1998.

18. Fotoohi M, D'Agostino HB, Wollman B, Chon K, Shahrokni S and vanSonnenberg E: Persistent pancreatocutaneous fistula after percutaneous drainage of pancreatic fluid collections: Role of cause and severity of pancreatitis. Radiology 213: 573-578, 1999. 
19. Baril NB, Ralls PW, Wren SM, Selby RR, Radin R, Parekh D, Jabbour N and Stain SC: Does an infected peripancreatic fluid collection or abscess mandate operation? Ann Surg 231: 361-367, 2000.

20. Bruennler T, Langgartner J, Lang S, Wrede CE, Klebl F, Zierhut S, Siebig S, Mandraka F, Rockmann F, Salzberger B, et al: Outcome of patients with acute, necrotizing pancreatitis requiring drainage-does drainage size matter? World J Gastroenterol 14 725-730, 2008.

21. Mortelé KJ, Girshman J, Szejnfeld D, Ashley SW, Erturk SM, Banks PA and Silverman SG: CT-guided percutaneous catheter drainage of acute necrotizing pancreatitis: Clinical experience and observations in patients with sterile and infected necrosis. AJR Am J Roentgenol 192: 110-116, 2009.

22. Rocha FG, Benoit E, Zinner MJ, Whang EE, Banks PA, Ashley SW and Mortele KJ: Impact of radiologic intervention on mortality in necrotizing pancreatitis: The role of organ failure. Arch Surg 144: 261-265, 2009.
23. van Grinsven J, van Dijk SM, Dijkgraaf MG, Boermeester MA, Bollen TL, Bruno MJ, van Brunschot S, Dejong $\mathrm{CH}$, van Eijck CH, van Lienden KP, et al; Dutch Pancreatitis Study Group: Postponed or immediate drainage of infected necrotizing pancreatitis (POINTER trial): Study protocol for a randomized controlled trial. Trials 20: 239, 2019.

24. Bansal A, Gupta P, Singh H, Samanta J, Mandavdhare H, Sharma V, Sinha SK, Dutta U and Kochhar R: Gastrointestinal complications in acute and chronic pancreatitis. JGH Open 3: 450-455, 2019.

25. Gupta P, Chayan Das G, Sharma V, Mandavdhare H, Samanta J, Singh H, Kant Sinha S, Dutta U and Kochhar R: Role of computed tomography in prediction of gastrointestinal fistula in patients with acute pancreatitis. Acta Gastroenterol Belg 82: 495-500, 2019.

26. Sikora SS, Khare R, Srikanth G, Kumar A, Saxena R and Kapoor VK: External pancreatic fistula as a sequel to management of acute severe necrotizing pancreatitis. Dig Surg 22: 446-451, discussion 452, 2005. 